\title{
Elementi di Ecografia in Emergenza-Urgenza
}

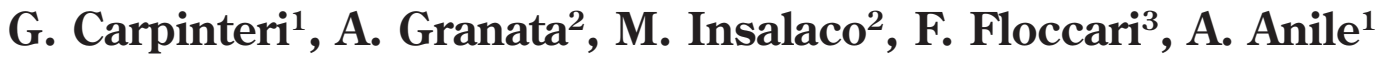 \\ ${ }^{1}$ U.O.C. di Medicina d’Urgenza e di Rianimazione, A.O.U. Policlinico "Vittorio Emanuele”, Catania \\ ${ }^{2}$ U.O.C. Nefrologia e Dialisi, Ospedale "San Giovanni di Dio", Agrigento \\ ${ }^{2}$ U.O.C. Nefrologia e Dialisi, Ospedale “San Paolo”, ASL RMF, Civitavecchia
}

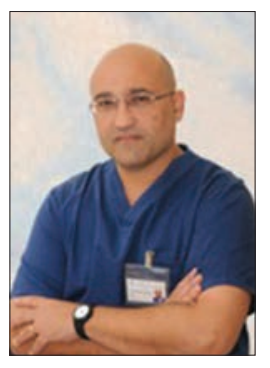

Giuseppe Carpinteri

\section{Introduzione}

Il setting dell'emergenza-urgenza richiede spesso un approccio clinico rapido e aggressivo a problematiche di vitale importanza, orientato dai segni e dai dati emodinamici, emogasanalitici, e laboratoristici a disposizione. In tale contesto la condotta terapeutica è fortemente indirizzata dall'intuito clinico e dalle informazioni derivanti dall'esame obiettivo (1). Luso della diagnostica per immagini è limitato da problematiche logistiche, dai costi umani e materiali e dalla tempistica di accesso alle prestazioni $(2,3)$.

Le metodiche di diagnostica ultrasonografica stanno registrando diffusione sempre maggiore in questo contesto, sempre più integrate nell'esame obiettivo di routine e sempre più direttamente operate dai Colleghi dell'emergenza-urgenza.

A differenza dell'ecografia in elezione, l'ecografia nelle emergenze/urgenze non è un'indagine panoramica, esplorativa, eseguita nel tentativo di escludere qualsiasi patologia diagnosticabile in quel determinato organo o regione anatomica. Essa è bensì focalizzata sul problema clinico e mira a ottenere rapidamente una serie di risposte semplici (di tipo sìno) ai quesiti che si presentano nel corso della valutazione dei pazienti (4). Esempi classici di quesiti a risposta rapida sono:

- È presente un tamponamento cardiaco in una situazione d'arresto?

- Il ventricolo sinistro si contrae normalmente?

- È presente dilatazione del ventricolo destro o trombosi venosa profonda nel sospetto di un'embolia polmonare?

- È presente aria nella cavità pleurica di un paziente con grave dispnea?
- Si può escludere una colecistite o un aneurisma aortico come cause di un dolore addominale?

Queste metodiche di approccio sintetico all'ecografia sono state codificate e identificate come FAST e FASTCRASH.

\section{La tecnica FAST}

L'ecografia in emergenza, finalizzata alla ricerca di raccolte ematiche all'interno del cavo peritoneale, pericardico o dello scavo pelvico nei pazienti traumatizzati, viene indicata con l'acronimo FAST (Focused Assessment with Sonography for Trauma) $(4,5)$. Questa tecnica d'esame, rigorosa ma rapida (5-6 minuti), prevede la scansione standardizzata di quattro aree:

- Periepatica (sottocostale sull'ascellare destra);

- Cardiaca sottodiaframmatica (sottoxifoidea);

- Perisplenica (sottocostale sull'ascellare sinistra);

- Pelvica.

Mediante tale tecnica si effettua una ricerca "bedside" di raccolte ematiche, offrendo una alternativa affidabile al lavaggio peritoneale o alla TC. L'utilizzo di tale approccio è divenuto praticamente capillare in alcuni contesti ad alta efficienza, come in gran parte del Nord America.

\section{La tecnica FAST-CRASH}

Recentemente è stata introdotta una variante detta FASTCRASH (dove CRASH sta per Cardiac arrest/failure, Respiratory arrest/failure, Acute Abdomen and Shock) che rappresenta un'estensione dell'approccio ecografico a tutte le emergenze mediche e chirurgiche rispetto a quelle solo traumatologiche considerate nella FAST (6).

La necessaria rapidità d'esecuzione e l'elevato impatto diagnostico esigono, per la FAST-CRASH, una semeiotica ecografica semplice e di qualità, che può essere limitata a tre segni fondamentali:

- Movimento alterato di strutture cardiache o pleuropol- 
monari (anomalie della cinetica ventricolare destra o sinistra nello shock o nella dispnea grave, assenza del "gliding sign" nel pneumotorace) o anomala risposta a manovre dinamiche di compressione eseguite con la sonda (ad esempio, non comprimibilità dei vasi venosi nella trombosi venosa profonda).

- Presenza di fluido in sedi anomale o in quantità anomala (ad esempio, nelle cavità sierose nel politrauma, all'interno dell'aorta con dilatazione del vaso nell'aneurisma, valutazione della distensione e variazioni respiratorie della vena cava inferiore, allinterno delle vie escretrici nell'idronefrosi, all'interno della colecisti con distensione del viscere nella colecistite acuta).

- Presenza di gas in sedi anomale (ad esempio, all'interno della cavità pleurica nel pneumotorace, all'interno della cavità pleurica nel pneumoperitoneo).

- L'approccio ecografico consente di distinguere in maniera affidabile lo shock cardiogeno da quello ipovolemico/distributivo e identificare la condizione di "polmone umido" nella dispnea severa. Esso sarà, inoltre, di grande utilità nel guidare le procedure invasive terapeutiche e nel monitorare la risposta agli interventi terapeutici (ad esempio, somministrazione di fluidi, supporto inotropo, trombolisi).

\section{L'ecografia nello studio del "polmone umido"}

Avere a disposizione un ecografo in DEA e UTI permette la valutazione dell'acqua extravascolare polmonare (EVLW) con tecniche di semplice acquisizione ed esecuzione, la cui accuratezza è molto alta $(7,8)$.

La valutazione della EVLW con ecografia polmonare si basa sulla presenza degli artefatti verticali a coda di cometa, a partenza dalla linea pleurica, detti linee B. Tali artefatti, se diffusi bilateralmente, sono segno della sindrome alveolo-interstiziale, manifestazione di alcune malattie acute e croniche polmonari, come l'edema cardiogeno, l'edema lesionale, la fibrosi polmonare e le polmoniti interstiziali $(9,10)$. Più in generale la presenza di linee B denota il quadro definito come "Polmone umido", che può indirizzare con elevata accuratezza nella diagnostica differenziale tra dispnee cardiogene e non cardiogene (11). Va ricordato che linee $\mathrm{B}$ sono presenti, in genere in numero inferiore a 8 , su entrambi i campi polmonari, anche nel polmone del soggetto normale. Le linee $B$ vengono generate dall'ampia differenza di impedenza acustica che si viene a produrre tra aria alveolare e setti interlobulari polmonari ispessiti dall'edema e corrispondono, pertanto, alle strie di Kerley evidenziabili alla radiografia del torace. La presenza di linee B identifica l'edema polmonare con valori di sensibilità prossimi al $100 \%$. La presenza di un pattern ecografico normale esclude di fatto la possibilità di un edema polmonare cardiogeno (12).

\section{L'ecografia nello studio della funzione cardiaca}

Uno studio ecografico completo della funzione cardiaca necessita di una sonda specifica, micro-convex, da 3 a 5 $\mathrm{MHz}$, e di un ecografo dotato di armonica tissutale.

In emergenza-urgenza l'approccio semplificato all'ecografia del cuore viene indicato come "ecocardioscopia" e può avvenire mediante una comune sonda macroconvessa, attraverso una valutazione sottocostale o apicale. L'approccio "apicale 4 camere" risulta essere la finestra ultrasonografica più ampia e intuitiva, perché il cuore sta a stretto contatto della parete toracica senza interposizione di tessuto polmonare e se ne ottiene una immagine ecografica di interpretazione immediata, anche ai non cardiologi.

Solitamente, l'itto cardiaco è identificabile all'ispezione o alla palpazione, con il paziente in decubito laterale sinistro e il trasduttore posizionato lateralmente e inferiormente rispetto all'itto, in modo da esaminare l'intero asse longitudinale del ventricolo sinistro.

I due ventricoli verranno quindi visualizzati nella porzione superiore dello schermo, mentre gli atri andranno a occuparne la porzione inferiore. Le camere sinistre appariranno sulla destra dello schermo, mentre quelle destre occuperanno la sinistra dello stesso.

Se dalla sezione " 4 camere" il trasduttore viene leggermente inclinato in avanti verso la parete anteriore del torace e ruotato in senso orario si visualizzerà il tratto di efflusso dal ventricolo sinistro e dal tratto prossimale dell'aorta compresa la valvola (" 5 camere"). L'immagine ricorda quella delle quattro camere, con in più la quinta camera (cioè l'aorta) disposta posteriormente tra i due atri.

Nell'approccio sotto-costale il paziente va esaminato in decubito supino e il trasduttore posto immediatamente al di sotto dell'apofisi xifoidea. Tale finestra acustica è così ampia da consentire l'utilizzo della sonda macroconvessa.

Va comunque ricordato che il software cardiologico residente sui comuni ecografi è progettato per essere utilizzato con la sonda micro-convex. L'utilizzo delle funzioni di calcolo più complesse, quali il calcolo dei volumi o della frazione di eiezione, su proiezioni ottenute da una sonda macro-convex comporta il rischio di ottenere risultati incongrui.

Ciò che è richiesto in urgenza è una valutazione veloce $\mathrm{e}$ solo qualitativa della performance cardiaca. Con un minimo training sarà possibile effettuare una vigilanza attiva delle condizioni emodinamiche del paziente, monitorando la contrattilità del ventricolo sinistro, escludendo la presenza di severa dilatazione delle camere destre (ad esempio, nel sospetto di embolia polmonare) o di versamento pericardico massivo con tamponamento (indicato 


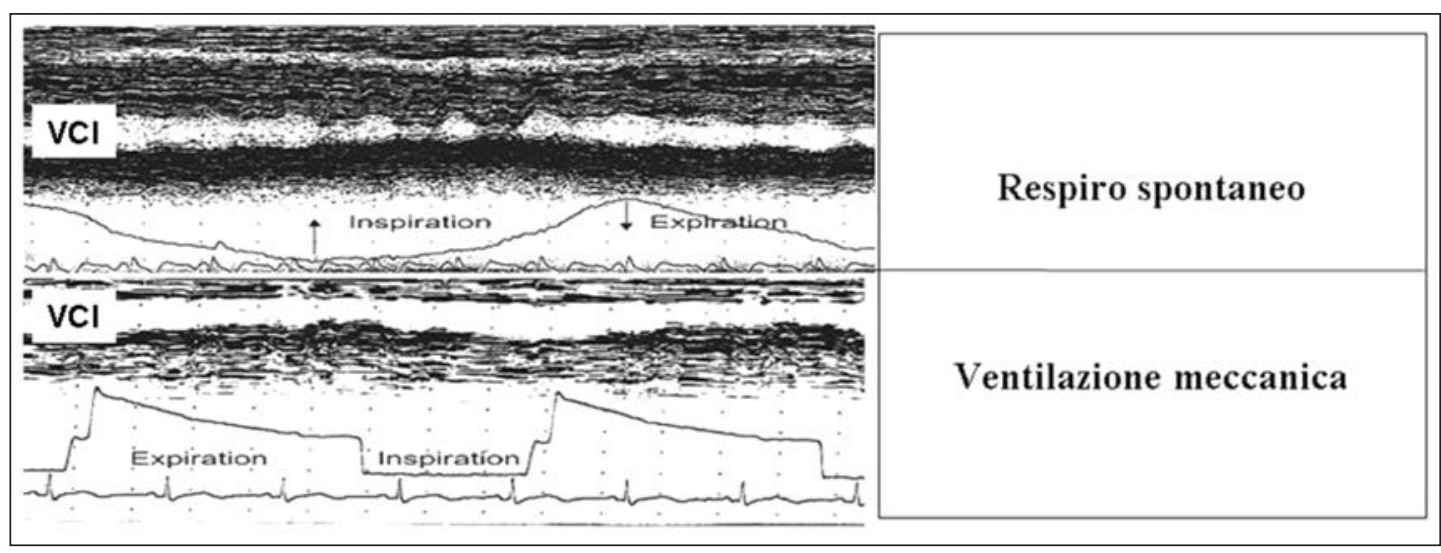

Fig. 1 - Variazioni inspiratorie ed espiratorie del diametro della VCI all'ecografia M-mode in respiro spontaneo $e$ in corso di ventilazione meccanica.

dal collasso telediastolico delle camere destre) o di masse intra-cardiache (13).

\section{La volemia}

La valutazione non invasiva dello stato volemico rappresenta una condizione essenziale per la gestione di un paziente instabile, soprattutto con funzionalità renale deficitaria. Una condizione di ipovolemia non corretta e una inappropriata infusione di inotropi può infatti condurre a una ipoperfusione d'organo e a ischemia (14). Di contro, una incongrua espansione volemica può sovraccaricando il circolo, determinare alterazioni respiratorie, incrementare l'ipossiemia e aumentare la pressione delle vie aeree, o può peggiorare una pre-esistente insufficienza cardiaca $(14,15)$.

La possibilità di predire la necessità e l'efficacia di una eventuale espansione volemica (fluid responsiveness) è condizione essenziale per la corretta gestione di un paziente gravemente compromesso. Per fluid responsiveness si intende la capacità di ricevere un incremento della gittata cardiaca in seguito ad espansione volemica. Essa cioè dipende da quale punto del tratto ascendente della curva di Frank-Starling in cui si trovi il miocardio in quel determinato momento clinico (15).

L'ecografia permette di visualizzare facilmente mediante un approccio sub-costale la vena cava inferiore (VCI). Questo è un vaso di capacitanza, la cui distensione è funzione diretta dello stato volemico $(16,17)$. Essa, origi- nando dalla fusione delle vene iliache di destra e di sinistra e terminando alla base dell'atrio di destra dopo aver attraversato il diaframma, è un vaso intra-addominale, la cui breve porzione intra-toracica è puramente virtuale. Nei pazienti in respiro spontaneo, i ciclici cambiamenti della pressione pleurica, trasmessi all'atrio destro, producono ciclici cambiamenti del ritorno venoso, inducendo un decremento inspiratorio del diametro della VCI e un aumento del suo diametro in fase espiratoria $(17,18)$. Linverso avviene nei pazienti ventilati meccanicamente, dove la fase inspiratoria produce un incremento della pressione pleurica, che viene trasmessa a livello atriale riducendo il ritorno venoso (Fig. 1).

I cambiamenti ciclici del diametro della VCI sono evidenti in condizioni di normale o ridotto stato volemico. Essi sono invece aboliti quando il vaso è dilatato a seguito di uno stato di ipervolemia e/o ipertensione atriale.

Kircher et al (19), hanno dimostrato che il decremento inspiratorio del diametro della VCI, espresso come indice, ossia

\section{Diametro espiratorio - diametro inspiratorio diametro espiratorio}

ben correla con la pressione atriale, nei pazienti in respiro spontaneo (Tab. I).

Risulta ragionevole pensare che in presenza di una VCI dilatata e immobile agli atti respiratori, sarà

TABELLA I - CORRELAZIONE TRA PVC, DIAMETRO VCI E SUA VARIABILITÀ INSPIRATORIA IN PAZIENTI IN RESPIRO SPONTANEO

Stima della pressione atriale $\mathrm{dx}$ o pressione venosa centrale (PVC)

Misura antero-posteriore

Riduzione inspiratoria

Collasso

$<1.5 \mathrm{~cm}$

$1.5-2 \mathrm{~cm}$

$1.5-2 \mathrm{~cm}$

$2-2.5 \mathrm{~cm}$

$>2.5 \mathrm{~cm}$
$>50 \%$

33-50\%

0-33\%

Assente
PVC

0-5 $\mathrm{mmHg}$

$5-10 \mathrm{mmHg}$

$10-15 \mathrm{mmHg}$

$15-20 \mathrm{mmHg}$

$>20 \mathrm{mmHg}$ 


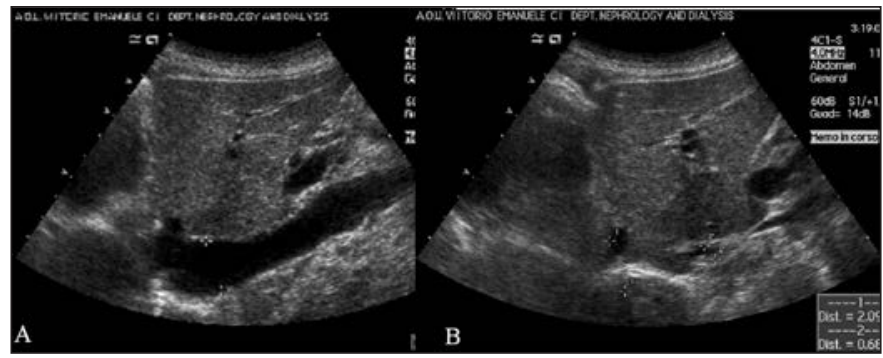

Fig. 2 - Ecografia B-mode, scansione longitudinale anteriore. A) Diametro VCI in espirazione $(2,09 \mathrm{~cm})$; B) Diametro VCI in inspirazione $(0,68 \mathrm{~cm})$. ICC $67 \%$ (pt normoidratato tendenzialmente ipovolemico).

probabilmente alta la PVC, escludendo quindi con buona certezza la presenza di ipovolemia e controindicando qualunque tentativo di espandere la volemia.

L'innalzamento passivo degli arti inferiori da $30^{\circ}$ a $45^{\circ}$ (Passive Leg Raising o PLR) è una manovra non invasiva che aggiunge, se correlata al contesto clinico del paziente, indicazioni circa lo stato volemico del paziente. Tale test, inducendo un aumento reversibile del ritorno venoso dagli arti inferiori al torace, comporta un aumento del flusso aortico o del volume di eiezione (20). Una variabilità respiratoria del diametro della VCI persistente dopo manovre di PLR escluderà uno stato ipervolemico, risultando indicativa di fluid responsiveness $(18,21)$.

La variabilità respiratoria della VCI è stata testata nella stima del "peso secco" in emodialisi, seppure con risultati controversi e non univoci. Già nel 1979, Natori et al (22) riportarono che la determinazione del diametro della VCI indicizzato (VCD) correlava con le pressioni atriale destre e con lo stato di idratazione del paziente. Altri Autori (23) utilizzarono tali misurazioni routinariamente nella pratica clinica, riconoscendo dei range di misurazioni entro i quali poter definire i pazienti dializzati iper-, normoo disidratati. Secondo tale orientamento, si definiva:

- "ipervolemico" un paziente con PVC superiore a $7 \mathrm{mmHg}$, un diametro indicizzato della VCI superiore a $11,5 \mathrm{~mm} / \mathrm{mq}$ e un indice di collassabilità (CI) minore del 40\%;

- "ipovolemico" un paziente con PVC inferiore a 3 $\mathrm{mmHg}$, correlato a un diametro indicizzato inferiore a $8 \mathrm{~mm} / \mathrm{mq}$ e un CI maggiore a $75 \%$.

Successivamente, altri Autori $(24,25)$, hanno confermato tali correlazioni, suggerendo però di escludere dall'utilizzo routinario i pazienti con grave compromissione della contrattilità cardiaca o con

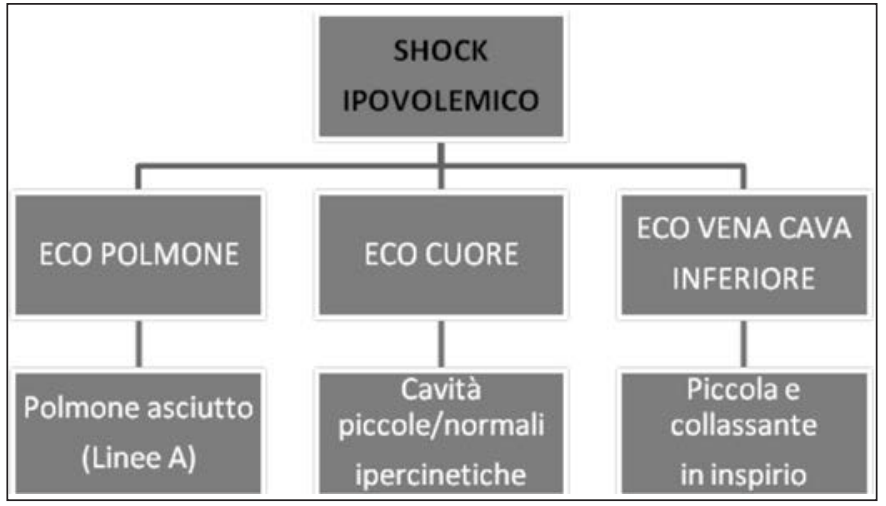

Fig. 3 - Schema semplificato per l'approccio al paziente con shock ipovolemico: rilievi ecografici.

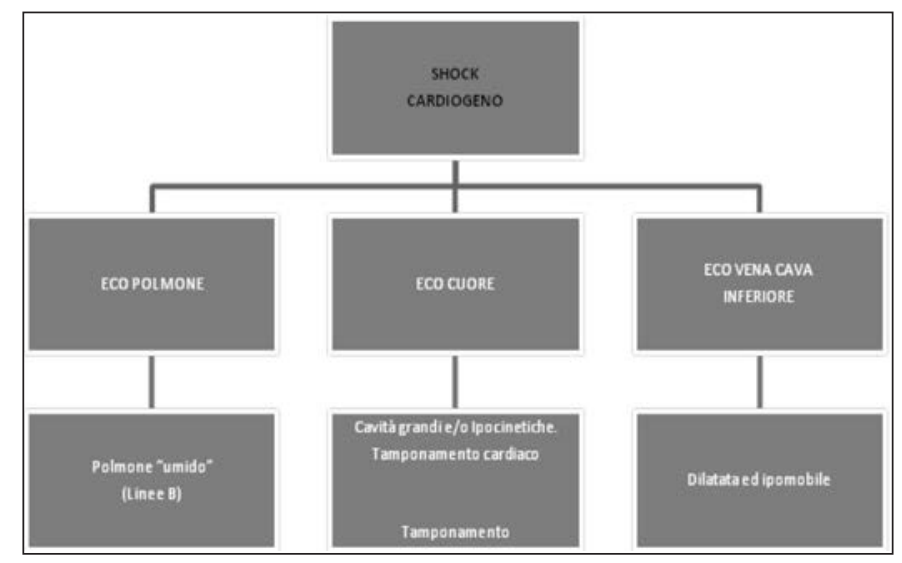

Fig. 4 - Schema semplificato per l'approccio al paziente con shock cardiogeno: rilievi ecografici.

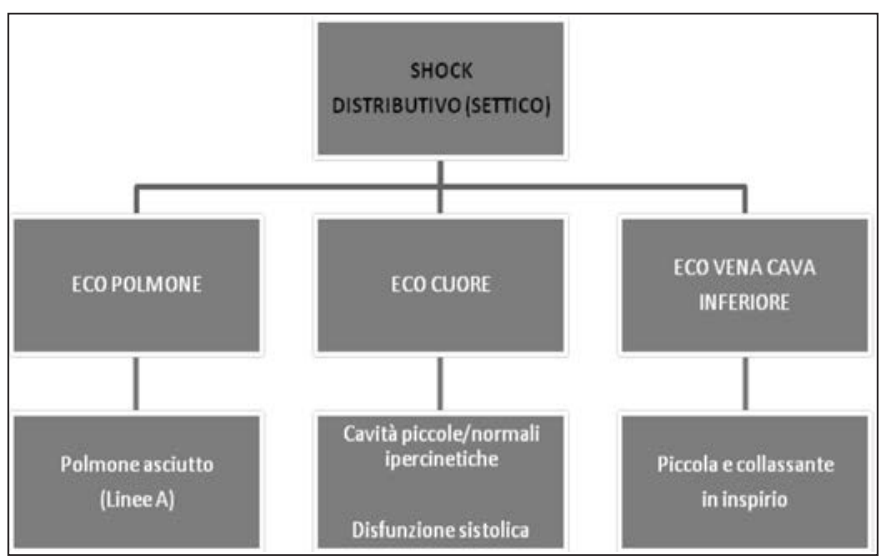

Fig. 5 - Schema semplificato per l'approccio al paziente con shock settico: rilievi ecografici.

severa insufficienza tricuspidale. Agarwal et al (14, 26), hanno invece dimostrato che se le variazioni respiratorie del diametro della VCI correlano efficacemente con le variazioni della volemia anche nei 
TABELLA II - PROPOSTA DI CHECK-LIST PER LA VALUTAZIONE CLINICA ECO GUIDATA

L'approccio prevede la risposta a precisi quesiti diagnostici tipo sì/no

\begin{tabular}{ll} 
Obiettivi dell'indagine ecografia & Quesiti \\
\hline Ventricolo dx & \\
& è piccolo? \\
& è grande? \\
& si muove?
\end{tabular}

Ventricolo sn

è piccolo? è grande? si muove?

Pericardio

c'è fluido? tampona?

Polmone

è asciutto? è umido?

Cavità pleuricalinea pleurica

c'è fluido?

Cavità peritoneale

c'è fluido?

Aorta addominale

è dilatata?

è rotta?

Vene arti inferiori

c'è TVP?

Vena cava inferiore

è di diametro aumentato? si muove col respiro?

dializzati, esse non risultano in alcuna misura predittive dell'espansione di volume intracellulare, una variabile di grande importanza nel paziente uremico (Fig. 2). Prudenzialmente occorre quindi sottolineare ancora una volta come la stima del peso secco rimanga il frutto di una analisi multiparametrica, strumentale, clinica e laboratoristica, e che a oggi non è ancora possibile ottenere risultati attendibili da una unica misurazione strumentale o laboratoristica $(27,28)$.

Al termine di questa breve e sintetica review, abbiamo voluto tracciare alcune tavole, riassuntive di quanto sin qui esposto. Seguiranno quindi una

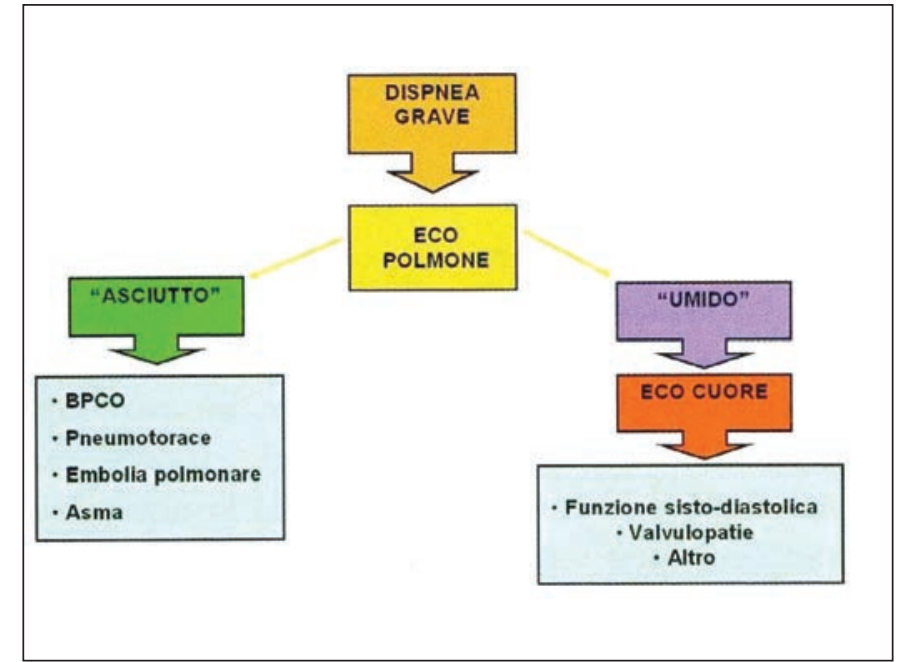

Fig. 6 - Schema semplificato per l'approccio al paziente con dispnea grave: rilievi ecografici.

check-list alla valutazione eco-guidata del paziente in condizioni di emergenza-urgenza (Tab. II) e delle flow-chart di approccio ecografico al paziente con shock (Figg. 3-6).

Indirizzo degli Autori:

Antonio Granata, MD

Via F. Paradiso 78/a

95024 Acireale (Catania)

antonio.granata4@tin.it

\section{Bibliografia}

1. Holley B, Vieillard-Baron A, Mebazaa A. Echocardiography in the ICU: time for widespread use! Intensive Care Med 2005; 16 November.

2. Kumar A, Anel R, Bunnell E, et al. Pulmonary artery occlusion pressure and central venous pressure fail to predict ventricular filling volume, cardiac performance, or the response to volume infusion in normal subjects. Crit Care Med 2004; 32: 691-9.

3. Squara P, Bennett D, Perret C. Pulmonary artery catheter: does the problem lie in the users? Chest 2002; 121: 2009-15.

4. American College of Emergency Physicians. ACEP emergency ultrasound guidelines 2001. Annals of Emergency Medicine 2001; 38(4): 470-81. 
5. Lichtenstein D, Lagoueyte JF, Gepner A, et al. A-lines and B-lines: lung ultrasound as a bedside tool for predicting pulmonary artery occlusion pressure in the critically ill. Chest 2009 36(4): 1014-20.

6. Cianci V. Ecografia in emergenza-urgenza. Torino: C.G. Edizioni Medico Scientifiche, 2005.

7. Lichtenstein D, Mezière G. A lung ultrasound sign allowing bedside distinction between pulmonary edema and CODP: the comet-tail artefact. Intensive Care Med 1998; 24(12): 1331-4.

8. Moore CL, Rose GA, Tayal VS, et al. Determination of left ventricular function by emergency physician echocardiography of hypotensive patients. Acad Emerg Med 2002; 9: $186-93$.

9. Randazzo MR, et al. Accuracy of emergency physician assessment of left ventricular ejection fraction and central venous pressure using echocardiography. Acad Emerg Med 2003; 10: 973-7.

10. Jambrik Z, Monti S, Coppola V, et al. Usefulness of ultrasound lung comets as a nonradiologic sign of extravascular lung water. Am J Cardiol 2004; 93: 1265-70.

11. Mallamaci F, Benedetto FA, Tripepi R, et al. Detection of pulmonary congestion by cest ultrasound in dialisi patients. J Am Coll Cardiol Img 2010; 3: 586-94.

12. Agricola E, Bove T, Oppizzi M, et al. Ultrasound comet tail images. A marker of pulmonary edema. Chest 2006; 127: 1690-5.

13. Wiedemann HP, Wheeler AP, Bernard GR, et al. Comparison of two fluid-management strategies in acute lung injury. N Engl J Med 2006; 354: 2564-75.

14. Agarwal R, Bouldin JM, Light RP, Garg A. Inferior vena cava diameter and left atrial diameter measure volume but not dry weight. Clin J Am Soc Nephrol 2011; 6: 1066-72.

15. Bendjelid K, Romand JA. Fluid responsiveness in mechanically ventilated patients: a review of indices used in intensive care. Intensive Care Med 2003; 29: 352-60.

16. Vieillard-Baron A, Prin S, Chergui K, et al. Hemodynamic instability in sepsis: bedside assessment by Doppler echocardiography. Am J Respir Crit Care Med 2003; 168: $1270-6$.
17. Fields JM, Lee PA, Jenq KY, Mark DG, Panebianco NL, Dean AJ. The Interrater Reliability of Inferior Vena Cava Ultrasound by Bedside Clinician Sonographers in Emergency Department Patients. Academic Emergency Medicine 2011; 18: 98-101.

18. Feissel M, Michard F, Faller JP, Teboul JL. The respiratory variation in inferior vena cava diameter as a guide to fluid therapy. Intensive Care Med 2004; 30:1834-7.

19. Kirker BJ, Himelman RB, Schiller NB.Non invasive estimation of right atrial pressure from the inspiratory collapse of the inferior vena cava. Am J Cardiol 1990; 66: 493-6.

20. Monnet X, Rienzo M, Osman D, et al. Passive leg raising predicts fluid resoponsiveness in the critically ill. Crit Care Med 2006; 34: 1402-07.

21. Pinsky MR, Payen D. Functional hemodynamic monitoring. Crit Care 2005; 9: 566-72.

22. Natori H, Tamaki S, Kira S. Ultrasonographic evaluation of ventilatory effect on inferior vena caval configuration. Am Rev Resp Dis 1979; 421-7.

23. Cheriex EC, Leunissen KM, Janssen JH, Mooy JM, van Hooff JP. Echography of the inferior vena cava is a simple and reliable tool for estimation of "dry weight" in haemodialysis patients. Nephrol Dial Transplant 1989; 4: 563-8.

24. Moreno FL, Hagan AD, Holmen JR, Pryor TA, Strickland $\mathrm{RD}$, Castle $\mathrm{CH}$. Evaluation of size and dynamics of the inferior vena cava as an index of right-sided cardiac function. Am J Cardiol 1984; 53: 579-85.

25. Krause I, Birk E, Davidovits M, et al. Inferior vena cava diameter: A useful method for estimation of fluid status in children on haemodialysis. Nephrol Dial Transplant 2001; 16: $1203-6$.

26. Haciomeroglu P, Ozkaya O, Gunal N, Baysal K. Venous collapsibility index changes in children on dialysis. Nephrology 2007; 12: 135-9.

27. Scuderi M. Ecografia Clinica nelle emergenze urgenze. Ed. Minerva Medica 2008.

28. Soldati G, Copetti R, Gargani L. Lung Ultrasonography for the cardiologist. G Ital Cardiol 2007; 8: 139-47. 